\title{
Iterative Autofocus Algorithms for Scanning Electron Microscopy
}

\author{
M. Rudnaya, R. M. M. Mattheij, and J. M. L. Maubach \\ Dept. of Mathematics and Computer Science, Eindhoven University of Technology, \\ PO Box 513, 5600 MB, Eindhoven, The Netherlands
}

A robust and reliable autofocus algorithm is important concern for the automation of a Scanning Electron Microscope (SEM). Comparison of existing autofocus techniques has been done for specific specimen for fluorescence [1] and non-fluorescence microscopy [2-3]. For Scanning Transmission Electron Microscopy some of available algorithms were compared [4]. To the authors' knowledge broad evolution has not been published yet for SEM.

We focus on iterative autofocus techniques with the use of a Sharpness Function (SF), a real-valued estimation of the sharpness of a discrete image. For the focus series SF should reach it's maxima for the in-focused image. Fig. 1 shows different SFs, as a function of the defocus, for the Gold-onCarbon sample focus series.

Existing SFs are based on the image gradient, variance, statistical information, histogram and Fourier Transform (FT). [1-3] evaluated the techniques, excluding the FT, because it required a high computational power that was not available several years ago. Nowadays we have enough computational power to use FT as a basis for SFs ([4] includes FT in their research). [1-3] consider gradient methods based on one parameter: The gray value threshold. We vary additional parameter the distance between compared pixels (as in [5]). Our evaluation shows, that with this parameter gradient-based SFs can show better results. For FT-based SF low and high frequency threshold are varying parameter.

The system of the SFs quality evaluation is based on the criteria in [1]. It considers accuracy (the difference in defocus steps between in-focused position and SF maximum), range (the interval where the SF is monotonically decreasing), amount of local maxima and Full Width and Half Maximum (FWHM) of a SF peak. Table 1 contains the results of evaluation of different SFs for Gold-on-Carbon focus series. For each criteria the value between 1 (the best) and 14 (the worst) was assigned. In our evaluation we show that the FT-based SFs appears to be one of the most reliable for SEM.

Focus series of different SEM samples obtained [6] in different modes with different noise levels are used for evaluation. We also consider SEM focus series with the effect of magnetic lens astigmatism. Due to the presence of astigmatism some of the SFs fail to reach the maxima in the infocused image, instead of this they demonstrate two maxima peaks in the neighborhood of the infocused position (see Fig. 1, Sq. Grad. plot) [7], [8].

\section{References}

[1] A. Santos et al., Jour. of Microsc. 188 (1997) 264.

[2] Y. Sun et al., Microsc. Res. and Techn. 65 (2004) 139.

[3] X. Y. Liu, W. H. Wang and Y. Sun, Jour. of Microsc. 227 (2007) 15. 
[4] W. Van den Broek, PhD thesis, University of Antwerp, 2007.

[5] J.F. Brenner et al., The Jour. of Histochem. and Cytochem. 24 (1976) 100.

[6] We kindly acknowledge R. van Vucht (FEI) for the automated recording of the focus series.

[7] We kindly acknowledge the helpful discussions with S. Sluyterman, R. van Vucht, E. Bosch, P.

Faber, M. Janus, M. Otten from FEI Company and W. Van den Broek (University of Antwerp).

[8] This work has been carried out as a part of the Condor project at FEI Company under the responsibilities of the Embedded Systems Institute (ESI). This project is partially supported by the Dutch Ministry of Economic Affairs under the BSIK program.

Table 1. An SF evaluation for a Gold-on-Carbon focus series, where the FT-based SF is ranked highest. Fig. 1 shows SFs for $\mathrm{N}=1,8,14$.

\begin{tabular}{llllllll}
\hline N & SF & Parameters & Accuracy & Range & Loc. Max & FWHM & Res. \\
\hline 1. & FT-based & High/Low freq.67, 21 & $0(1)$ & $27(1)$ & $0(1)$ & $5.4(6)$ & 9 \\
2. & Sq. Gradient & Thresh.,dif. 100, 101 & $0(1)$ & $27(1)$ & $0(1)$ & $5.7(7)$ & 10 \\
3. & Abs. Gradient & Thresh.,dif. 100,101 & $0(1)$ & $27(1)$ & $0(1)$ & $6.1(8)$ & 11 \\
4. & Sq. Gradient & Thresh.,dif. 0, 101 & $0(1)$ & $27(1)$ & $0(1)$ & $8.7(9)$ & 12 \\
5. & Variance & - & $0(1)$ & $27(1)$ & $0(1)$ & $8.9(10)$ & 13 \\
6. & Vollah (SDBC) & - & $0(1)$ & $27(1)$ & $0(1)$ & $9(11)$ & 14 \\
7. & Vollah(ACR,XY) & - & $1(9)$ & $27(1)$ & $0(1)$ & $4.6(3)$ & 14 \\
8. & Enthropy & - & $0(1)$ & $27(1)$ & $0(1)$ & $18.3(13)$ & 16 \\
9. & Sq. Gradient & Thresh.,dif. 0,1 & $1(9)$ & $26(9)$ & $1(9)$ & $4.5(2)$ & 29 \\
10. & Vollah (ACR, X) & - & $1(9)$ & $25(11)$ & $1(9)$ & $3.4(1)$ & 30 \\
11. & Abs. Gradient & Thresh.,dif. 0,1 & $1(9)$ & $26(10)$ & $1(9)$ & $5.3(5)$ & 33 \\
12. & FT, non-threshold & - & $1(9)$ & $10(13)$ & $4(14)$ & $5(4)$ & 40 \\
13. & Range & - & $0(1)$ & $6(14)$ & $3(13)$ & $22.4(14)$ & 42 \\
14. & Sq. Gradient & Thresh.,dif. 100,1 & $2(14)$ & $17(12)$ & $1(9)$ & $9.3(12)$ & 47 \\
\hline
\end{tabular}

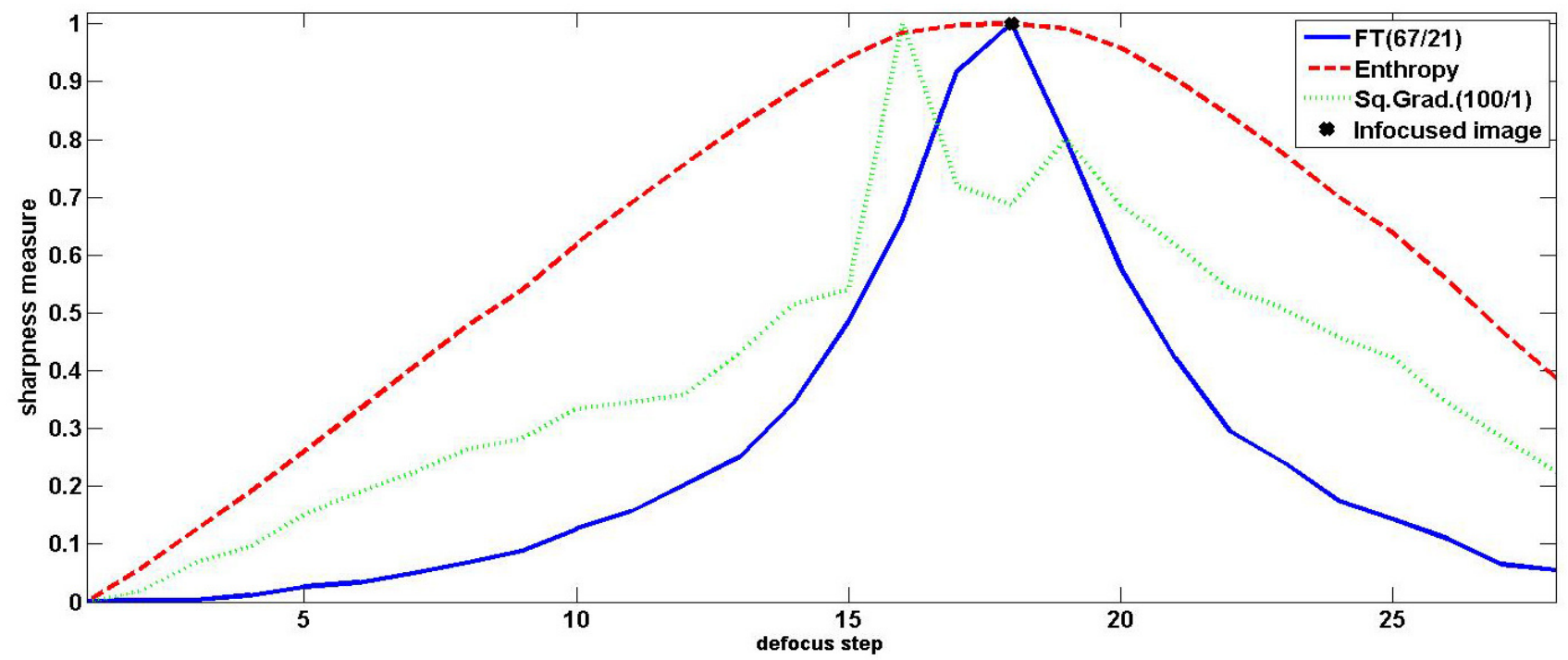

Fig. 1. Three SF for the Gold-on-Carbon focus series plotted versus defocus. 